\title{
Tim
} JORNAL INTERDISCIPLINAR DE BIOCIÊNCIAS

Homepage: http://www.ojs.ufpi.br/index.php/jibi

\section{Os efeitos da atividade física na remodelação óssea}

The effects of physical activity on bone remodeling

\author{
Karinn de Araújo Soares ${ }^{1 *}$ e Mozart Bastos Oliveira Filho \\ ${ }^{1}$ Departamento de Morfologia, Universidade Federal do Piauí; ${ }^{2}$ Faculdade Santo Agostinho/Faculdade Maurício de Nassau
}

\begin{abstract}
A B S T R A C T
Bone is a dynamic tissue, developed and adapted by hormones, cytokines and mechanical stimuli, such as physical activity. The bone undergoes changes, even after the end of skeletal growth, comprising the remodeling process, that is a balance between apposition and bone reabsorption. In this context, the maintenance of bone mineral density (BMD) is very important for the prevention of metabolic bone diseases, such as osteoporosis, which is characterized by a marked decrease in BMD. Studies indicate that physical activity is positively related to BMD, being an important factor for its maintenance. Among these studies, some use force training as an intervention, in an attempt to increase the BMD of individuals submitted to this type of physical activity. At the cellular level, the process of remodeling induced by overload in physical activity, seems to be performed by the action of osteocytes, which act as mechanical receptors of the applied stress, and release a chemical stimulating factor of the proliferation of osteoblasts in the stressed place. This study aimed to evaluate the effect of physical activity on bone remodeling. For this purpose, information was searched in the databases of CAPES, MEDLINE, LILACS and in the SciELO virtual library, selecting papers published in national and international periodicals between 1999 and 2016. The descriptors of subjects used were: "physical activity "," Bone remodeling "," bone mineral density ", using the intersection of sets. Although some results are contradictory, the literature does not question the beneficial effects of physical activity on bone tissue, both in healthy individuals, and in the prevention and treatment of Osteoporosis. The mechanisms by which physical activity stimulates osteoblastic differentiation from bone marrow stromal cells should be better elucidated so that more appropriate therapies can be developed and used in the treatment of individuals with some metabolic bone disease.
\end{abstract}

KE Y W OR D S

Motor activity, Bone remodeling, Bone density

R E S M O

O osso é um tecido dinâmico, desenvolvido e adaptado por hormônios, citocinas e estímulos mecânicos, como a atividade física. Ele sofre alterações, mesmo após o final do crescimento do esqueleto, compreendendo o processo de remodelação, ou seja, um equilíbrio entre a posição e reabsorção óssea. Nesse contexto, a manutenção da densidade mineral óssea (DMO) é muito importante para a prevenção de doenças ósseas metabólicas, como a osteoporose, a qual é caracterizada por uma diminuição acentuada da DMO. Estudos indicam que a atividade física está positivamente relacionada com a DMO, sendo um importante fator para sua manutenção. Dentre estes estudos, alguns utilizam o treinamento de força como intervenção, na tentativa de aumentar a DMO de indivíduos submetidos a esse tipo de atividade física. Em nível celular, o processo de remodelação, induzido pela sobrecarga, como na atividade física, parece ser realizado pela ação dos osteócitos, que atuam como receptores mecânicos do estresse aplicado e liberam um fator químico estimulador da proliferação de osteoblastos no local estressado. Este trabalho teve como objetivo avaliar o efeito da atividade física na remodelação óssea. Para isso, buscaram-se informações nas bases de dados da CAPES, MEDLINE, LILACS e na biblioteca virtual SciELO, selecionando-se artigos publicados em periódicos nacionais e internacionais entre os anos de 1999e 2016. Os descritores de assunto utilizados foram: "atividade física", "remodelação óssea", "densidade mineral óssea", utilizando a interseção dos conjuntos. Embora alguns resultados sejam contraditórios, a literatura não deixa dúvidas quanto aos efeitos benéficos da atividade física sobreo tecido ósseo, tanto em indivíduos saudáveis, quanto na prevenção e tratamento da osteoporose. Os mecanismos pelos quais a atividade física estimula a diferenciação osteoblástica, a partir das células do estroma da medula óssea, devem ser melhores elucidados, para que terapêuticas mais adequadas possam ser desenvolvidas e utilizadas no tratamento de indivíduos acometidos com alguma doença óssea metabólica.

PALA VRAS - CHAVE

Atividade física, Remodelação óssea, Densidade óssea 


\section{INTRODUÇÃO}

O osso é um tecido conjuntivo mineralizado que confere estrutura de suporte altamente especializada ao corpo, além de apresentar rigidez, dureza, poder de regeneração e de reparo, proteger os órgãos vitais, proporcionar um ambiente para medula óssea, na formação sanguínea e no armazenamento de gordura, e participar da realização do movimento. Esse tecido constantemente sofre remodelação durante a vida, para se adaptar à evolução das forças biomecânicas, ao envelhecimento e a micro danos ósseos, até ser substituído por um osso novo, mecanicamente mais forte (TAICHMAN, 2005; DATTA et al., 2008; KINI; NANDEESH, 2012; REDLICH; SMOLEN, 2012; REGARD et al., 2012).

Uma vez que o crescimento do esqueleto está completo por volta dos vinte anos de idade, a saúde dos ossos é mantida pelos processos acoplados de reabsorção do osso pré-existente por osteoclastos e de formação de osso novo por osteoblastos. A coordenação desses processos é denominada de remodelação óssea e ocorre ao longo da vida, exigindo uma constante comunicação entre osteoclastos, osteócitos e osteoblastos, para que haja equilíbrio entre os processos de reabsorção e formação ósseas e, consequente, manutenção da densidade mineral óssea (DMO) (MORAES et al., 2002; RAISZ, 2005; NARDONE et al., 2014).

A enorme variação na DMO é explicada não só por fatores hereditários, como também por sexo, raça, hábitos dietéticos, influências hormonais, composição corporal, doenças intercorrentes, uso crônico de medicamentos e, inclusive, por atividade física. Nesse contexto, Brandão e Vieira (1999) afirmam que se o ganho mineral ósseo puder ser otimizado durante a puberdade, é provável que o indivíduo adulto esteja menos susceptível a sofrer as devastadoras complicações de doenças metabólicas ósseas, como a osteoporose.

Este estudo teve como objetivo avaliar o efeito da atividade física na remodelação óssea. Para isso, as informações foram buscadas nas bases de dados da CAPES, MEDLINE, LILACS e na biblioteca virtual SciELO. Foram selecionados artigos publicados em periódicos nacionais e internacionais, entre os anos de 1999 e 2016. Os descritores de assunto utilizados foram: "atividade física", "remodelação óssea", "densidade mineral óssea", utilizando a interseção dos conjuntos.

\section{DESENVOLVIMENTO}

Macroscopicamente, o osso maduro é classificado em cortical ou compacto, o qual possui maior densidade de matriz óssea e trabecular, ou esponjoso, quando apresenta cavidades intercomunicantes visíveis, onde se encontra a medula óssea (HING, 2004; RUFFONI et al., 2008). Microscopicamente, o osso apresenta componentes celulares e os componentes da matriz. Dentre as células associadas ao tecido ósseo, três tipos são exclusivos: osteoblastos, osteócitos e osteoclastos, responsáveis, respectivamente, pela síntese, manutenção e reabsorção do tecido (HING, 2004; RUFFONI et al., 2008).

O osso é um tecido dinâmico, desenvolvido e adaptado por hormônios, citocinas e fatores externos, como estímulos mecânicos (MAENO et al., 2013). Além disso, o osso sofre alterações, mesmo após o final do crescimento do esqueleto, compreendendo o processo de remodelação, ou seja, um equilíbrio entre a aposição e a reabsorção óssea (REAL et al., 2009).

Nesse contexto, a manutenção da DMO é muito importante para a prevenção de doenças ósseas metabólicas, como a osteoporose, caracterizadas por uma diminuição acentuada da DMO (VINCENT; BRAITH, 2002), na qual a matriz e os minerais ósseos são perdidos devido ao excesso de reabsorção óssea em relação à formação, sendo um processo normalmente associado ao avanço da idade e à ocorrência da menopausa (MAIMOUN et al., 2003).

Embora a perda óssea seja mais intensa nas mulheres (HUMPHRIES et al., 2000; MAIMOUN et al., 2003), os homens também apresentam uma diminuição da DMO devido à idade avançada (RYAN et al., 2004).

Vários estudos indicam que a atividade física está positivamente relacionada com a DMO, sendo um importante fator para a sua manutenção (MAIMOUN et al., 2003). É consensual, na literatura especializada, que atividades físicas de maior sobrecarga, decorrente do peso corporal, bem como o treinamento de força, causam estímulos osteogênicos, devido ao aumento do estresse mecânico localizado nos ossos (CREIGHTON et al., 2001; LIU et al., 2003). A realização de atividade física é recomendada devido à possibilidade de potencializar o pico de massa óssea. Desse modo, o aumento da massa magra é o fator preditor mais importante para a acumulação de massa mineral óssea, durante o crescimento (FERREIRA et al., 2015).

Estudos indicam que a atividade física está positivamente relacionada com a DMO, sendo um importante fator para sua manutenção (KEMMLER et al., 2002; MAIMOUN et al., 2003; OCARINO; SERAKIDES, 2006). Entre esses estudos, alguns utilizam o treinamento de força como intervenção, na tentativa de aumentar a DMO de indivíduos submetidos a esse tipo de atividade física (HUMPHRIES et al., 2000). Além disso, crianças que praticam esporte, durante o crescimento, incrementam o pico de DMO entre $10 \%$ a $20 \%$, quando comparado aos pares que não praticam, sendo que o período mais sensível, no crescimento, para o aumento da densidade óssea é entre 
11-13 anos para meninas e 12-14 anos para meninos (VICENTE-RODRÍGUEZ, 2006).

Nesses casos, o aumento na DMO deve-se à estimulação da formação óssea e não à atenuação da reabsorção que ocorre nesse tecido. Em nível celular, o processo de remodelação, induzido pela sobrecarga, como na atividade física, parece ser realizado pela ação dos osteócitos, que atuam como receptores mecânicos do estresse aplicado e liberam um fator químico estimulador da proliferação de osteoblastos no local estressado (ANDREOLI et al., 2001).

Uma possível justificativa para o aumento da DMO com o treinamento de força é o efeito piezoelétrico ósseo. A força mecânica, quando aplicada sobre o tecido ósseo, forma sinais endógenos que interferem nos processos de remodelação óssea. Esses sinais são captados por um sistema mecanossensorial, no qual o osteócito é a principal célula responsável por traduzir a força mecânica em sinais bioquímicos que regulam o turnover ósseo (NOMURA; YAMAMOTO, 2000; CHERIAN et al., 2003). Acredita-se que a deformação celular causada pela força direta sobre a célula, o aumento da pressão intracanalicular provocado pela força dinâmica e o incremento da velocidade do fluxo do fluido intersticial sejam fatores que afetam diretamente o osteócito, sendo esse último o maior estímulo à referida célula, em resposta à carga mecânica. Dessa forma, o fluxo de fluido intersticial pelos canalículos, ao redor do osteócito, parece ser responsável pela deformação da matriz extracelular e por alterações nas membranas celulares (CHERIAN et al., 2003). A atividade física também promove aumento das conexões das ramificações canaliculares dos osteócitos, aumentando a viabilidade da matriz óssea (OCARINO, 2004).

Em estudo de caso-controle, comparando meninos com e sem fraturas de antebraço em relação à DMO, consumo diário de produtos derivados de leite e atividade física, Pires e colaboradores (2005) afirmam que, apesar das contribuições genéticas para a DMO, os fatores ambientais, incluindo exercícios físicos, são importantes, especialmente antes da puberdade. Os mesmos autores sugerem que o exercício físico possa ter um reflexo positivo sobre a massa óssea, sendo maior nas pessoas que praticam exercício.

A influência mecânica sobre o osso se aplica diretamente ao funcionamento fisiológico normal deste e o crescimento longitudinal é controlado por fatores mecânicos locais sob a forma de um mecanismo de feedback, que existe para garantir que o osso cresça na direção desejada (FERREIRA et al., 2015).

Em estudo de Silva e colaboradores (2015), testou-se a relação de força e nível de atividade física com a DMO de mulheres na pós-menopausa. Observou-se que as mulheres que não praticaram atividade física, de sobrecarga óssea de moderada à alta, na fase da adolescência até a idade adulta, tiveram risco aumentado em cerca de seis vezes de apresentarem diminuição da DMO em comparação às mulheres com DMO normal, consolidando a importância da atividade física.

A determinação de qual o tipo de atividade física seja a ideal para aumentar o pico de massa óssea na adolescência, ou mesmo mantê-la após a idade adulta, é muito importante para a prevenção e o possível tratamento da osteoporose, cuja incidência ocorre principalmente em mulheres pósmenopáusicas (MAIMOUN et al., 2003). Além disso, as associações da DMO com a força muscular e a composição corporal sugerem que a prescrição de um treinamento, que vise melhorar esses parâmetros, pode ter um efeito benéfico na DMO (CADORE et al., 2005).

\section{CONCLUSÃO}

Embora fatores como genética, homeostase e hormonal e alimentação possam ser determinantes na DMO, o nível de atividade física assume importante influência nessa variável. Apesar do mecanismo fisiológico não ser inteiramente claro, a ação osteogênica da atividade física parece ser mediada via efeito piezoelétricoósseo.

Mesmo encontrando alguns resultados contraditórios, a literatura não deixa dúvidas quanto aos efeitos benéficos da atividade física sobre o tecido ósseo, tanto em indivíduos saudáveis, quanto na prevenção e tratamento de doenças ósseas metabólicas. Os mecanismos pelos quais a atividade física estimula a diferenciação osteoblástica, a partir das células do estroma da medula óssea, devem ser melhor elucidados, para que terapêuticas mais adequadas possam ser desenvolvidas e utilizadas no tratamento de indivíduos com osteoporose, principalmente daqueles que possuem restrições para a prática de atividade física ou de mulheres na pós-menopausa.

Desta forma, mais investigações devem ser realizadas para verificar a relação dos diferentes tipos de prática de atividade física, volume e intensidade de treinamento com a maturação, crescimento e desenvolvimento humano.

\section{REFERÊNCIAS}

ANDREOLI, A.; MONTELEONE, M.; VAN LOAN, M.; PROMENZIO, L.; TARANTINO, U.; DE LORENZO, A. Effects of different sports on bone density and muscle mass in highly trained athletes. Med. Sci. Sports Exerc, v. 33, p. 507-511, 2001.

BRANDÃO, C. M. A.; VIEIRA, J.G.H. Fatores Envolvidos no Pico de Massa Óssea. Arq. Bras. Endocrinol. Metab, v. 43, n. 6, p. 401-408, 1999.

CADORE, E.L.; BRENTANO, M. A.; KRUEL, L. F. M. Efeitos da atividade física na densidade mineral óssea e na remodelação do tecido ósseo. R. Bras. Med. Esporte, v. 11, n. 6, nov/dez, 2005. 
CHERIAN, P. P.; CHENG, B.; GU, S.; SPRAGUE, E. BONEWALD, L.F.; JIANG, J.X. Effects of mechanical strain on the function of gap junctions in osteocytes are mediated through the prostaglandin EP2 receptor. J. Biol. Chem, v. 278, n. 44, p. 43146-43156, 2003.

CREIGHTON, D. L.; MORGAN, A. L.; BOARDLEY, D.; BROLINSON, P. G. Weight-bearing exercise and markers of bone turnover in female athletes. J. Appl. Physiol, v. 90, n. 2, p. 565-570, 2001.

DATTA, H. K.; NG, N. F.; WALKER, J. A.; TUCK, S. P.; VARANASI, S. S. The cell biology of bone metabolism. J. Clin. Pathol, v. 61, n. 5, p. 577-587, 2008.

FERREIRA, M. N. G.; MATEUS, J. R.; MATEUS, S.; COSTA, G. C. T. A influência da atividade física e esportes sobre o crescimento e a maturação. Revista Brasileira de Futsal e Futebol, Ed. Supl. 1, v.7, n.24, p.237-243. 2015.

HING, K. A. Bone repair in the twenty-first century: biology, chemistry or engineering? Philos. Trans. A. Math. Phys. Eng. Sci. v. 362, n. 1825 , p. 2821-50, dec. 2004.

HUMPHRIES, B.; NEWTON, R. U.; BRONKS, R.; MARSHALL, S.; MCBRIDE, J.; MCBRIDE, T. T. Effect of exercise intensity on bone density, strength, and calcium turnover in older women. Med. Sci. Sports Exerc, v. 32, n. 6, p. 1043 1050, 2000.

KEMMLER, W.; ENGELKE, K.; LAUBER, D.; WEINECK, J.; HENSEN, J.; KALENDER, W.A. Exercise effects on fitness and bone mineral density in early post-menopausal women: 1-year EFOPS results. Med. Sci. Sports Exerc, v. 34, n. 12, p. 21152123, 2002.

KINI, U.; NANDEESH, B.N. Physiology of bone formation, remodeling and metabolism. In: Fogelman et al. (eds.) Radionuclide and hybrid bone imaging. Berlin: SpringerVerlag, 2012. 1046 p. p. 29-57.

LIU, L.; MARUNO, R.; MASHIMO, T.; SANKA, K.; HIGUCHI, T.; HAYASHI, K. Effects of physical training on cortical bone at midtibia assessed by peripheral QCT. J. Appl. Physiol, v. 95, n. 1, p. 219-24, 2003.

MAENO, M.; TANAKA, H.; ZHANG, F.; KITAMI, S.; NAKAI, K.; KAWATO, T. Direct and indirect effects of IL-17A on RANKL-induced osteoclastogenesis. J. Hard. Tissue Biol, v. 22, n. 3, p. 287-292, 2013.

MAIMOUN, L.; LUMBROSO, S.; MANETTA, J.; PARIS, F.; LEROUX, J.L.; SULTAN, C. Testosterone is significantly reduced in endurance athletes without impact on bone mineral density. Horm. Res, v. 59, n. 6, p. 285-92, 2003.

MORAES, F.F.; CHAVES, V.E.A.; FAVA, M. Fundamentos histológicos aplicados à ortodontia. In: INTERLANDI S. Ortodontia: bases para iniciação. 5. ed. São Paulo: Artes Médicas, 2002. p. 89-100.

NARDONE, V.; D'ASTA, F.; BRANDI, M. L. Pharmacological management of osteogenesis. Clinics, v. 69, n. 6, p. 438-446, 2014.
NOMURA, S.; YAMAMOTO, T. T. Molecular event caused by mechanical stress in bone. Matrix Biol., v. 19, n. 2, p. 91-96, 2000 .

OCARINO, N. M. Atividade física no tratamento da osteoporose: histomorfometria do esqueleto axial e apendicular de ratas. Belo Horizonte, 2004. - Dissertação (Mestrado em Medicina Veterinária). Belo Horizonte, Escola de Veterinária, UFMG, 2004. 78p.

OCARINO, N. M.; SERAKIDES, R. Efeito da atividade física no osso normal e na prevenção e tratamento da osteoporose. R. Bras. Med. Esporte, v. 12, n. 3, mai/jun, 2006.

PIRES, L. A. S.; SOUZA, A. C. A.; LAITANO, O.; MEYER, F. Densidade mineral óssea, ingestão de leite e atividade física de meninos que sofreram fraturas no antebraço. J. Pediatr., v. 81, n. 4, p. 332-6, 2005.

RAISZ, L. G. Pathogenesis of osteoporosis: concepts, conflicts, and prospects. J. Clin. Invest, v. 115, n. 12, p. 3318-3325, dec. 2005 .

REAL, L. A. S. V.; RAMOS, A. L.; ZANONI, J. N. Modificações no periodonto de ratos diabéticos após movimentação ortodôntica. R. Dental Press Ortodon. Ortop Facial, v. 14, n. 1, p. 124-131, jan./fev. 2009.

REDLICH, K.; SMOLEN, J. S. Inflammatory bone loss: pathogenesis and therapeutic intervention.. Nat. Rev. Drug Discov. v. 11, n. 3, p. 234-250, mar. 2012.

REGARD, J. B.; ZHONG, Z.; WILLIAMS, B. O.; YANG, Y. Wnt signaling in bone development and disease: making stronger bone with Wnts.. Cold. Spring Harb. Perspect. Biol, v. 4, n. 12, dec. 2012.

RUFFONI, D.; FRATZL, P.; ROSCHGER, P.; PHIPPS, R.; KLAUSHOFER, K.; WEINKAMER, R. Effect of temporal changes in bone Turnover on the bone mineralization density distribution: a computer simulation study. J. Bone Min. Res, v. 23, n. 12, p. 1905-1914, 2008.

RYAN, A. S.; IVEY, F. M.; HURLUBUT, D. E.; MARTEL, G. F.; LEMMER, J. T.; SORKIN, J. D. Regional bone mineral density after resistive training in young and older men and women. J. Appl. Physiol.,v. 14, n. 1, p. 16-23, 2004.

SILVA, C. F. F.; AMORIM, P. R. S.; CARVALHO, C. J.; FARIA, M. M.; LIMA, L. M. Associação de força e nível de atividade física à densidade mineral óssea na pós-menopausa. $\mathbf{R}$. Bras. Med. Esporte, v. 21, n.2. mar/abr, 2015.

TAICHMAN, R. S. Blood and bone: two tissues whose fates are intertwined to create the hematopoietic stem-cell niche. Blood, v. 105, n. 7, p. 2631-2639, apr. 2005.

VICENTE-RODRÍGUEZ, G. How does exercise affect bone development during growth? Sports Medicine, v. 36, n. 7, p.561$569,2006$.

VINCENT, K. R.; BRAITH, R. W. Resistance training and bone turnover in elderly men and women. Med. Sci. Sports Exerc, v. 34, n. 1 , p. $17-23,2002$ 